Rev. Adm. Saúde (On-line), São Paulo, v. 21, n. 83: e295, abr. - jun. 2021, Epub 28 mai. 2021 http://dx.doi.org/10.23973/ras.83.295

PERSPECTIVAS

\title{
Dividindo a experiência de gestão médica no maior serviço privado de urgência pediátrica do país
}

Sharing the experience of medical management in the largest private pediatric emergency service in the country

\section{Felipe Monti Lora ${ }^{1}$}

\section{RESUMO}

O autor relata a sua experiência de oito anos (2013-2021) a frente da gestão médica do pronto-socorro do Sabará Hospital Infantil, São Paulo SP, maior serviço privado de urgência pediátrica do país. O gestor, neste período, enfrentou excessiva demanda de atendimento com insatisfação de pacientes, dificuldades para gerir equipes desmotivadas por pobre modelo de atendimento e de remuneração. Foram formuladas ações, em um planejamento de cinco anos, baseadas na: a) integração pelo conhecimento não técnico, ensinando ao profissional como o mercado o valoriza; b) predileção pela qualidade em grupo: simbolizada pela conquista da acreditação Joint Commission International; c) atratividade competitiva: equiparação de honorários com mercado e remuneração por mérito; e d) desenvolvimento de novo conceito de produtividade médica com o comprometimento do profissional com a eficiência operacional. As ações levaram ao aumento na velocidade de atendimento da equipe entre $15 \%$ e $25 \%$, dependendo do mês. Houve um salto de honorários na ordem de $15 \%$ a $100 \%$ dependendo da faixa. Foi possível reduzir o desperdício de horas médicas da escala em 7,4\%. O engajamento relacionado a protocolos clínicos se estabeleceu, e a equipe contava com $60 \%$ mais pediatras. No quinquênio que se seguiu, dificuldades colocaram à prova os 
valores desenvolvidos pela equipe, sendo a principal relacionada à pandemia do covid-19, com a redução abrupta do volume de atendimento. Como resposta houve redução de horas de escala em $45 \%$, com garantia de honorários médicos mensais mínimo de $65 \%$ e desligamento de apenas 10\% dos profissionais. Ao fim do quinquênio foram criados projetos de melhorias que hoje diferenciam a instituição, tais como: a) Supervia (2019) - protocolo para atendimento de casos menos urgentes que permite alta logo após a triagem, reduzindo o tempo de espera do paciente; b) Telemedicina (2020) - além das consultas de urgência, utilizada também na reavaliação de pacientes; c) Short stay (2021) - espaço destinado a internações de baixa complexidade com previsão de duração inferior a 48 horas, observada uma lista de vinte diagnósticos selecionados.

Palavras-chave: Organização e administração de serviços de saúde; Remuneração médica, Carga de trabalho; Pediatria; Pandemia de covid-19

\begin{abstract}
The author reports his experience of eight years (2013-2021) in front of the medical management of the emergency room at Sabará Hospital Infantil, São Paulo SP, the largest private pediatric emergency service in the country. The manager, in this period, faced excessive demand for care with patient dissatisfaction, difficulties to manage teams unmotivated by a poor service and remuneration model. Actions were formulated in a five-year plan based on: a) integration through non-technical knowledge, teaching the professional how the market values it; b) predilection for group quality: symbolized by the achievement of Joint Commission International accreditation; c) competitive attractiveness: matching fees with the market and merit pay; and d) development of a new concept of medical productivity with the professional's commitment to operational efficiency. The actions led to an increase in the team's service speed between $15 \%$ and $25 \%$, depending on the month. There was a jump in fees in the order of $15 \%$ to $100 \%$ depending on the range. It was possible to reduce the waste of medical hours on the scale by $7.4 \%$.

Engagement related to clinical protocols was established, and the team had $60 \%$ more pediatricians. In the five years that followed, difficulties put to the test the values developed by the team, the main one being related to the covid-19 pandemic, with the abrupt reduction in the volume of care. In response, there was a $45 \%$ reduction in hours of work, with a minimum monthly medical fee of $65 \%$ guaranteed and dismissal of only $10 \%$ of professionals. At the end of the five-year period, improvement projects were created that today differentiate the institution, such as: a) Supervia (2019) - protocol for the treatment of less urgent cases that allows discharge immediately after the screening, reducing the patient's waiting time; b) Telemedicine (2020) - in addition to emergency consultations, also used to reassess patients; c) Short stay (2021) - space destined to hospitalizations of low complexity with a prediction of less than 48 hours, observing a list of twenty selected diagnoses.
\end{abstract}

Keywords: Organization and administration of health services; Medical fees; Workload; Pediatrics; Covid-19 pandemic 


\section{Introdução}

Nos últimos oito anos à frente do pronto-socorro do Sabará Hospital Infantil (PS), aprendi lições valorosas para dividir. Não por se tratar de um período longo, mas porque foi um período de muitas mudanças nos direcionamentos da estratégia do hospital, no mercado de urgência pediátrica no que tange os tipos de demanda do paciente e sua relação com as diferentes mídias, assim como àquelas mais especificamente relacionadas a pandemia covid-19.

Todo este cenário trouxe desafios e oportunidades para a gestão do Serviço de Urgência do Sabará, tanto para a manutenção do que não poderia mudar, quanto para a agilidade na transformação daquilo que não deveria continuar "como sempre foi".

Encontrar um método que levasse a identificação do que deveria ser mantido e do que deveria ser transformado foi o principal desafio. E este relato tem como objetivo compartilhar esta experiência.

\section{Ambientação}

Em junho de 2013, os serviços pediátricos de urgência em São Paulo encontravam-se com excessiva demanda quantitativa de atendimento e tempo de espera pela consulta médica de até seis horas, tendo este sido assunto de matérias nos jornais.

Qualquer serviço apresenta maior dificuldade em gerir equipes quando sobrecarregado. Médicos desmotivados no ambiente de trabalho naturalmente estressante da emergência hospitalar, sendo o plantão seu segundo ou terceiro emprego, apenas renda complementar, por vezes, de modo temporário, formavam a condição perfeita para um alto turn over, baixo engajamento profissional e falta de modelo de atendimento que pudesse trazer qualidade assistencial além daquela relacionada a competência médica individual.

Havia um verdadeiro "rouba-monte" de pediatras, conforme eram oferecidos efêmeros diferenciais que faziam parte do convite de trabalho (em ordem crescente de atratividade): valor monetário adicional por ficha de paciente atendido, melhor valor por hora de plantão, melhores períodos na escala de trabalho, regime de contratação CLT.

O Sabará Hospital Infantil contava com o mesmo número de pediatras de outros serviços, apesar de atender volume mensal de crianças três vezes superior e, portanto, precisar cobrir carga horária de plantões muito maior. Para compensar, o número de horas médicas na escala era 8,3\% acima do necessário (ineficiência esperada quando se tem doze profissionais 
trabalhando simultaneamente de modo liberal e liberado). Com remuneração em torno de $15 \%$ abaixo do mercado, tínhamos $10 \%$ das horas médicas na escala desfalcadas e conseguíamos suprir apenas metade das horas adicionais necessárias em momentos de pico. Como consequência observávamos um índice elevado de insatisfação relacionada à espera do público atendido, motivo este responsável por mais de dois terços das reclamações.

\section{Horizonte}

Após minha apresentação formal pela instituição como novo gerente do prontosocorro e breve período de observação, fizemos a primeira reunião de equipe. Esse momento foi um marco na história da unidade pois fundamentou um planejamento de cinco anos, o qual se baseava nos seguintes pilares táticos:

- Integração pelo conhecimento não técnico: ao perguntar o que era "pacote" de receita (forma predominante de remuneração em PS), menos de $5 \%$ dos médicos levantaram a mão. Não se tratava de influenciar a conduta clínica, mas de integrar a equipe à unidade. Ensinar como o mercado o valoriza, era respeitar o profissional e fazê-lo pertencer ao serviço.

- Predileção pela qualidade em grupo: naquele momento, simbolizada pela conquista da acreditação Joint Commission International, a qualidade precisava ser embasada na independência do perfil de coordenação. Lembro-me de dizer "as mudanças que definirmos juntos devem ficar na equipe mesmo depois que eu sair um dia". Isso sobrepunha as necessidades da equipe às individuais, fomentando o espírito de time.

- Atratividade competitiva: equiparação de honorários com mercado e proposta de futura variação de remuneração por mérito.

- Desenvolvimento de novo conceito de produtividade médica - exposição voluntária da equipe que provasse o comprometimento do setor com a eficiência operacional e traria brio ao setor.

Este último pilar tático gerou questionamentos maiores e desencadeou formalização de discussões por WhatsApp. O nome do grupo (ainda em uso) tem sentido duplo: "PS rules". Define o local de alinhamento de regras que seguimos. Mas também resgata a autoestima do setor pois remete à expressão inglesa que significa PS "no comando".

\section{Giro de rota}

A produtividade não era uma novidade como método para aumentar a eficiência do atendimento médico, reconhecidamente o principal gargalo na fila 
de pronto-socorro. Qualquer serviço que pague seus profissionais por hora e tenha a receita por evento tenderá a querer aumentar o volume de atendimentos por período. Todavia, na saúde há que se observar o aspecto ético e o risco na qualidade assistencial, se associado a um atendimento médico demasiadamente rápido.

A produtividade nas urgências - e não era diferente ali - garantia um valor fixo em torno de $80-90 \%$ dos honorários médicos. O restante, variável, era a soma de pequenos valores pagos por pacientes atendidos. Ocorre que, ao final de um plantão, a variação de honorários entre aqueles que mais e menos atendiam era muito pequena, não valendo o "sacrifício". Mas os cálculos gerenciais mostravam que esse esforço isoladamente já seria capaz de reduzir a fila de pacientes em $30 \%$.

Assim, a produtividade passou a representar um fator de accountability da equipe. Permitia a seleção de pessoas com o perfil de fazer seu melhor, ou seja, de maior engajamento na nova mentalidade da organização do PS. Sua simbologia era maior que os números potenciais, o que facilitou sua implantação.

O primeiro passo foi colocar freio ao excesso: a recomendação do Conselho Federal de Medicina de três pacientes atendidos por hora em média, para nós seria o máximo.

Então, todas atividades do plantão - não apenas o atendimento - foram consideradas: períodos de descanso, refeições, ritmo menor do atendimento infantil, quantidade histórica de reavaliações... para chegarmos a números que seriam o ideal e o mínimo aceitáveis em velocidade de atendimento.

Com isso - e talvez aqui esteja o "pulo do gato" - foram desenvolvidas faixas de produtividade. No decorrer do mês, eram somados (de modo ponderado por risco) os pacientes atendidos por cada profissional e dividido por suas horas de trabalho. Esse cálculo, retroativo, mostrou dispersão histórica, que permitiu estabelecer faixas onde todos plantonistas pudessem melhorar e atingir, aumentando assim seus honorários.

Agora cada atendimento não contava mais como um pequeno valor naquele plantão, mas contribuía para - ao fim da jornada mensal - um salto de honorários na ordem de $15 \%$ por faixa. Desse modo, os honorários entre as faixas extremas variavam em até $100 \%$.

A ação levou ao aumento na velocidade de atendimento média da equipe em $15 \%$ já no primeiro mês, chegando a culminar na época de pico em $25 \%$ mais veloz. Números estes bastante expressivos para um setor historicamente pressionado a ser eficiente.

Em um ano o PS se estabilizava, com escala quase completa e cobertura de $90 \%$ das horas adicionais liberadas. Foi possível reduzir o desperdício de horas médicas da escala em 7,4\% devido a maior rapidez no atendimento. Algum engajamento qualitativo relacionado a protocolos clínicos já se esboçava e a equipe contava com $60 \%$ mais pediatras. 
A produtividade foi a primeira vitória conjunta e mudou os olhos da alta direção do hospital a respeito do pronto-socorro, que deixava de ser o "patinho feio" (financeiramente deficitário) e passava a ter reconhecidos os $80 \%$ de dependência das internações que traziam receita ao Hospital.

\section{Turbulências}

No quinquênio que se seguiu, dificuldades colocaram à prova os valores desenvolvidos pela equipe. Todas as ações de gestão consideraram necessidades médicas individuais contrapesadas de maneira transparente com as necessidades do serviço como um todo. A soma desses elementos culminava em equilíbrio que ia ficando cada vez mais estável e se ancorava no propósito da unidade. Este, motivo comum a ser defendido.

A tabela abaixo resume os desafios com as medidas que denotam esses valores.

\begin{tabular}{|l|l|l|l|l|}
\hline Ano & Desafio & $\begin{array}{l}\text { Impactos na } \\
\text { equipe }\end{array}$ & Solução & Desfechos \\
\hline 2015 & $\begin{array}{l}\text { Diretriz de } \\
\text { redução } \\
\text { excessiva de } \\
\text { horas médicas } \\
\text { em momento } \\
\text { que precedia o } \\
\text { pico sazonal }\end{array}$ & $\begin{array}{l}\text { Abandono de } \\
\text { pediatras do } \\
\text { serviço (-30\%) }\end{array}$ & $\begin{array}{l}\text { Aumento de 100\% } \\
\text { do custo da hora } \\
\text { médica (valor de } \\
\text { plantão dobrado) } \\
\text { dispensa voluntária } \\
\text { remunerada em } \\
\text { meses de baixo } \\
\text { movimento e bônus } \\
\text { financeiro no final do } \\
\text { ano relacionado ao } \\
\text { mérito } \\
\text { (produtividade e } \\
\text { responsabilidade por } \\
\text { horas de escala) }\end{array}$ & $\begin{array}{l}\text { Retorno do custo } \\
\text { médico ao valor } \\
\text { de mercado }\end{array}$ \\
$\begin{array}{l}\text { Estabilização de } \\
\text { escala (<4 meses) }\end{array}$ & $\begin{array}{l}\text { Aumento de } \\
\text { equipe (+78\%) }\end{array}$ \\
\hline $\begin{array}{l}\text { Troca de } \\
\text { direção técnica } \\
\text { do hospital }\end{array}$ & $\begin{array}{l}\text { Reclassificação de } \\
\text { atribuições } \\
\text { médicas e } \\
\text { coordenações }\end{array}$ & $\begin{array}{l}\text { Implantação de } \\
\text { medidas teóricas } \\
\text { gestão }\end{array}$ & $\begin{array}{l}\text { Participação } \\
\text { médicos junto à } \\
\text { gestão, fora do } \\
\text { horário de escala }\end{array}$ \\
$\begin{array}{l}\text { Desenvolvimento de } \\
\text { novas reuniões } \\
\text { administrativas de } \\
\text { benchmarking } \\
\text { rotina mensal }\end{array}$ & $\begin{array}{l}\text { Amadurecimento } \\
\text { de equipe rumo } \\
\text { à versatilidade } \\
\text { resolutiva }\end{array}$ \\
\hline
\end{tabular}




\begin{tabular}{|c|c|c|c|c|}
\hline $\begin{array}{l}2017- \\
2019\end{array}$ & $\begin{array}{l}\text { Capacidade de } \\
\text { atendimento } \\
\text { do hospital } \\
\text { suplantada pela } \\
\text { demanda de } \\
\text { pacientes } \\
\text { graves }\end{array}$ & $\begin{array}{l}\text { Acúmulo de } \\
\text { pacientes } \\
\text { internados no } \\
\text { setor de urgência } \\
\text { Fechamento da } \\
\text { porta do PS para } \\
\text { atendimentos que } \\
\text { não de emergência }\end{array}$ & $\begin{array}{l}\text { Reuniões dos } \\
\text { diversos setores do } \\
\text { hospital para } \\
\text { soluções conjuntas: } \\
\text { realocação de } \\
\text { colaboradores, giro } \\
\text { de leitos, mudanças } \\
\text { de fluxo e } \\
\text { incremento } \\
\text { estrutural ágil para } \\
\text { cuidar dos internados } \\
\text { (+130\% de leitos no } \\
\text { PS) }\end{array}$ & $\begin{array}{l}\text { Plano de } \\
\text { Contingência, } \\
\text { que dá } \\
\text { previsibilidade e } \\
\text { transparência } \\
\text { nas ações de pico } \\
\text { - hoje, um } \\
\text { documento } \\
\text { alinhado com a } \\
\text { missão } \\
\text { institucional }\end{array}$ \\
\hline 2020 & $\begin{array}{l}\text { Pandemia } \\
\text { covid-19: } \\
\text { redução } \\
\text { abrupta do } \\
\text { volume de } \\
\text { atendimento }\end{array}$ & $\begin{array}{l}\text { Redução em horas } \\
\text { de escala } \\
\text { Redução de } \\
\text { pessoas } \\
\text { Adaptação a novos } \\
\text { fluxos de } \\
\text { isolamento }\end{array}$ & $\begin{array}{l}\text { Plano “55/85" - } \\
\text { redução de horas } \\
\text { escala em } 45 \% \text {, e } \\
\text { rodízio que garantia } \\
\text { honorários médicos } \\
\text { mensais (65-100\%) } \\
\text { "Plano 60+" - } \\
\text { pediatras mais } \\
\text { experientes atuando } \\
\text { em telemedicina } \\
\text { (70\% de honorários } \\
\text { garantidos) }\end{array}$ & 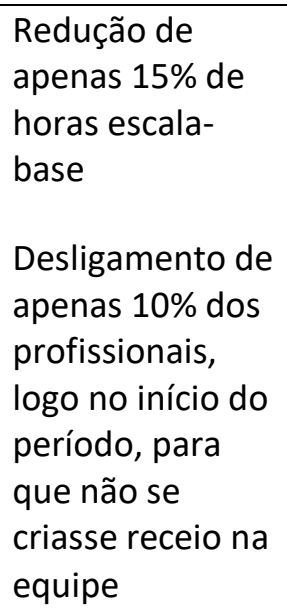 \\
\hline
\end{tabular}

\section{Novos ventos}

O intuito de ter uma equipe forte não é apenas resistir às intempéries. $A$ versatilidade desenvolvida, alicerçada na confiança, nos permitiu criar projetos de melhorias que hoje nos diferenciam, tais como:

- Supervia (2019): protocolo para atendimento de casos menos urgentes que permite alta logo após a triagem, reduzindo o tempo de espera dos pacientes. Através de uma seleção de condições clínicas relativamente simples, o enfermeiro pode direcionar o paciente para esse "by-pass", onde o uso de recursos é muito baixo ou nenhum. O projeto parte do princípio de que a retirada da fila daqueles pacientes que requerem consultas mais objetivas, agiliza o atendimento dos pacientes mais graves que requerem mais tempo médico. O paciente da Supervia tem sua espera quase nula, enquanto o paciente do fluxo habitual também é beneficiado.

- Telemedicina (2020) - além das consultas de urgência, utilizada também na reavaliação de pacientes, com follow up no mesmo dia em alguns casos selecionados. Conforme vontade do paciente e possibilidade 
clínica, a reavaliação médica não precisa ser feita no PS. Exemplos são resultados de exames de urina em paciente já examinado e sem sinais de gravidade, teste rápido de influenza ou estreptococo em orofaringe.

- Short stay (2021) - espaço destinado a internações de baixa complexidade com previsão de duração inferior a 48 horas. Observada uma lista de vinte diagnósticos em alguns hospitais americanos de modelo Children's, selecionamos e customizamos para a realidade de nosso PS. Assim, possibilitamos continuidade do cuidado pela equipe do pronto-socorro em internações curtas. Trata-se de casos que não se resolvem nas seis horas de PS, mas em ambiente de internação comum poderiam acabar ficando mais que o necessário. Laringite ou urticária gigante que não melhoram nas primeiras quatro horas são exemplos claros que quase todo médico de urgência já vivenciou!

\section{Conclusão: a dinâmica}

Se pudermos resumir os princípios que embasaram o modus operandi da equipe do pronto-socorro do Sabará em uma fórmula, capaz de formar um time tão competitivo, ela seria:

$\begin{array}{lll}\text { (Necessidade das pessoas }+ & \text { Alinhamento a estratégia empresa) } \\ \text { Cultura gerada }= & \times & \begin{array}{l}\text { Valores do setor } \\ \text { p/ objetivo } \\ \text { maior }\end{array}\end{array}$

Adaptação ao ambiente

\section{REFERÊNCIAS}

1. Sociedade Brasileira de Pediatria [internet]. Longa espera vira rotina para crianças em hospitais particulares de SP. 22 abril 2013.

https://www.sbp.com.br/imprensa/detalhe/nid/longa-espera-vira-rotina-paracriancas-em-hospitais-particulares-de-sp/

2. Estadão [internet]. Novas práticas e telemedicina garantem segurança para pacientes em hospital infantil. 26 maio 2020.

https://saude.estadao.com.br/noticias/geral,novas-praticas-e-telemedicinagarantem-seguranca-para-pacientes-em-hospital-infantil,70003314782 
3. Hopper S M, Archer P, Breene R, Bolt P, Sammartino L. Paediatric short stay unit in a community hospital: effective, efficient and popular. Emerg Med Australas. 2008 Oct;20(5):431-6.

https://pubmed.ncbi.nlm.nih.gov/18973641/

Recebido: 12 de maio de 2021. Aceito: 28 de maio de 2021

Correspondência: Felipe Monti Lora. E-mail: felipemlora@hotmail.com

Conflito de Interesses: o autor declarara não haver conflito de interesses

(C) This is an Open Access article distributed under the terms of the Creative Commons Attribution License, which permits unrestricted use, distribution, and reproduction in any medium, provided the original work is properly cited 